Pacific Journal of Mathematics

ON GENERAL Z.P.I.-RINGS 


\section{ON GENERAL Z.P.I.-RINGS}

\section{CraIg A. WoOD}

A commutative ring in which each ideal can be expressed as a finite product of prime ideals is called a general Z.P.I.ring (for Zerlegungsatz in Primideale). A general Z.P.I.-ring in which each proper ideal can be uniquely expressed as a finite product of prime ideals is called a Z.P.I.-ring. Such rings occupy a central position in multiplicative ideal theory. In case $R$ is a domain with identity, it is clear that $R$ is a Dedekind domain ${ }^{1}$ and the ideal theory of $R$ is well known. If $R$ is a domain without identity, the following result of Gilmer gives a somewhat less known characterization of $R$ : If $D$ is an integral domain without identity in which each ideal is a finite product of prime ideals, then each nonzero ideal of $D$ is principal and is a power of $D$; the converse also holds. Also somewhat less known is the characterization of a general Z.P.I.-ring with identity as a finite direct sum of Dedekind domains and special primary rings. ${ }^{2}$

This paper considers the one remaining case: $R$ is a general Z.P.I.-ring with zero divisors and without identity. A characterization of such rings is given in Theorem 2. This result is already contained ih a more obscure form in a paper by $\mathrm{S}$. Mori. The main contribution here is in the directness of the approach as contrasted to that of Mori.

In order to prove Theorem 2 we need to establish two basic properties of a general Z.P.I.-ring $R: R$ is Noetherian and primary ideals of $R$ are prime powers. Having established these two properties of $R$, the following result of Butts and Gilmer in [3], which we label as (BG), is applicable and easily yields our characterization of general Z.P.I.-rings without identity.

(BG), [3; Ths. 13 and 14]: If $R$ is a commutative ring such that $R \neq R^{2}$ and such that every ideal in $R$ is an intersection of a finite number of prime power ideals, then $R=F_{1} \oplus \cdots \oplus F_{k} \oplus T$ where each $F_{i}$ is a field and $T$ is a nonzero ring without identity in which every nonzero ideal is a power of $T$.

It is important to note that we do not use Butts and Gilmer's

1 M. Sono [14] and E. Noether [13] were among the first to consider Dedekind domains. For a historical development of the theory of Dedekind domains see [4; pp. 31-32].

${ }^{2} \mathrm{~S}$. Mori in [11] considered both general Z.P.I.-rings with identity and Z.P.I.rings without identity which contain no proper zero divisors, but Mori's results in these cases are not as sharp as those of Asano and Gilmer. 
paper [3] to prove that a general Z.P.I.-ring is Noetherian, while Butts and Gilmer do use this result from Mori's paper [11; Th. 7]. Theorem 2 gives a finite direct sum characterization of a general Z.P.I.-ring whereas Theorems 3 and 4 and Corollary 2 give characterizations of a general Z.P.I.-ring in terms of ideal-theoretic conditions.

Since we are only concerned with commutative rings, "ring" will always mean "commutative ring". The notation and terminology is that of [16] with two exceptions: $\subseteq$ denotes containment and $\subset$ denotes proper containment, and we do not assume that a Noetherian ring contains an identity. If $A$ is an ideal of a ring $R$, we say that $A$ is a prper ideal of $R$ if $(0) \subset A \subset R$ and that $A$ is a genuine ideal of $R$ if $A \subset R$.

2. Structure theorem of a general Z.P.I.-ring. In this section section we prove directly that a general Z.P.I.-ring is Noetherian by proving that each of its prime ideals is finitely generated. We then use result (BG) to prove the structure theorem of a general Z.P.I.ring.

Definition. Let $R$ be a ring. If there exists a chain $P_{0} \subset P_{1} \subset \cdots \subset P_{n}$ of $n+1$ prime ideals of $R$ where $P_{n} \subset R$, but no such chain of $n+2$ prime ideals, then we say that $R$ has dimension $n$ and we write $\operatorname{dim} R=n$.

Lemma 1. If $R$ is a general Z.P.I.-ring, $R$ contains only finitely many minimal prime ideals and $\operatorname{dim} R \leqq 1$.

Proof. If $R$ contains no proper prime ideal, then the lemma is clearly true. Therefore, we assume $R$ contains a proper prime ideal $P$ and we show that $R$ contains a minimal prime ideal. If $P$ is not a minimal prime of $R$, there exists a prime ideal $P_{1}$ such that $P_{1} \subset P \subset R$. It follows that $R / P_{1}$ is a domain containing a proper prime ideal in which each ideal can be represented as the product of finitely many prime ideals. This implies that $R / P_{1}$ is a Dedekind domain [6]. Therefore, $P_{1}$ is a minimal prime of $R$. This also shows that $\operatorname{dim} R \leqq 1$.

Since $R$ is a general Z.P.I.-ring, there exist prime ideals $Q_{1}, \cdots, Q_{n}$ in $R$ and positive integers $e_{1}, \cdots, e_{n}$ such that $(0)=$ $Q_{1}^{e_{1}} \cdots Q_{n}^{e_{n}}$. If $M$ is a minimal prime ideal of $R,(0)=Q_{1}^{e_{1}} \cdots Q_{n}^{e_{n}} \cong M$ which implies that $Q_{i} \subseteq M$ for some $i$. Hence, $M=Q_{i}$ and it follows that the collection $\left\{Q_{1}, \cdots, Q_{n}\right\}$ contains all the minimal prime ideals of $R$. Therefore, $R$ contains only finitely many minimal prime ideals.

Lemma 2. If $R$ is a general Z.P.I.-ring containing a genuine 
prime ideal, then each minimal prime ideal of $R$ is finitely generated.

Proof. ${ }^{3} \quad$ Let $P$ be a minimal prime ideal of $R$ and let $\left\{P_{1}, \cdots, P_{n}\right\}$ be the collection of minimal primes of $R$ distinct from $P$. If $P=(0)$, the proof is clear. If $(0) \subset P$, we show that $P$ is finitely generated by an inductive argument; that is, we show how to select a finite number of elements in $P$ which generate $P$. We divide the proof into three cases.

Case 1. $P=P^{2}$. Since $P=P^{2} \cong R P \sqsubseteq P, P=R P$. Now,

$$
P \nsubseteq \bigcup_{i=1}^{n} P_{i}
$$

since $P \nsubseteq P_{i}$ for $1 \leqq i \leqq n$ so let $\mathrm{x}_{1} \in P \backslash\left(\bigcup_{i=1}^{n} P_{i}\right)$. Thus, there exist prime ideals $M_{1}, \cdots, M_{s}$, positive integers $e_{0}, e_{1}, \cdots, e_{s}$, and a nonnegative integer $e_{s+1}$ such that

$$
\left(x_{1}\right)=P^{e_{0}} M_{1}^{e_{1}} \cdots M_{s}^{e_{s}} R^{e_{s+1}}=P M_{1}^{e_{1}} \cdots M_{s}^{e_{s}} R^{e_{s+1}}=P M_{1}^{e_{1}} \cdots M_{s}^{e_{s}}
$$

since $P=R P$. Let $\delta=\sum_{i=1}^{s} e_{i}$. If $P=\left(x_{1}\right)$, we are done. If $\left(x_{1}\right) \subset P$, then by choice of $x_{1}$ each $M_{i}$ is a maximal prime ideal of $R$. Then [2; Proposition 2, p. 70] implies that $P \nsubseteq\left\{\left(x_{1}\right) \cup\left(\bigcup_{i=1}^{n} P_{i}\right)\right\}$. If $x_{2} \in P \backslash\left\{\left(x_{1}\right) \cup\left(\bigcup_{i=1}^{n} P_{i}\right)\right\}$, then

$$
\left(x_{2}\right)=P M_{1}^{f_{1}} \cdots M_{s}^{f_{s}} R^{f_{s+1}} Q_{1}^{g_{1}} \cdots Q_{t}^{g_{t}}=P M_{1}^{f_{1}} \cdots M_{s}^{f_{s}} Q_{1}^{g_{1}} \cdots Q_{t}^{g t}
$$

where $Q_{i}$ is a maximal prime ideal of $R$ for $1 \leqq j \leqq t, f_{i} \in \omega_{0}$ for $1 \leqq i \leqq s+1$, and $g_{j} \in w$ for $1 \leqq j \leqq t$. Since $\left(x_{2}\right) \nsubseteq\left(x_{1}\right)$, we have that $e_{i_{0}}>f_{i_{0}}$ for some $i_{0}, 1 \leqq i_{0} \leqq s$. Therefore,

$$
\begin{aligned}
\left(x_{1}, x_{2}\right) & =P M_{1}^{e_{1}} \cdots M_{s}^{e_{s}}+P M_{1}^{f_{1}} \cdots M_{s}^{f_{s}} Q_{1}^{g_{1}} \cdots Q_{t}^{g_{t}} \\
& =P M_{1}^{m_{1}} \cdots M_{s}^{m_{s}}\left(M_{1}^{e_{1}-m_{1}} \cdots M_{s}^{e_{s}-m_{s}}+M_{1}^{f_{1}-m_{1}} \cdots M_{s}^{f_{s}-m_{s}} Q_{1}^{g_{1}} \cdots Q_{t}^{g_{t}}\right)
\end{aligned}
$$

where $m_{i}=\min \left\{e_{i}, f_{i}\right\}$ for $1 \leqq i \leqq s$. By the definition of $m_{i}$, if $e_{i}-m_{i} \neq 0$, then $f_{i}-m_{i}=0$, and if $f_{i}-m_{i} \neq 0$, then $e_{i}-m_{i}=0$. Let $A=M_{1}^{e_{1}-m_{1}} \cdots M_{s}^{e_{s}-m_{s}}$ and let $B=M_{1}^{f_{1}-m_{1}} \cdots M_{s}^{f_{s}-m_{s}} Q_{1}^{g_{1}} \cdots Q_{t}^{g_{t}}$, we show that $A+B$ is contained in no maximal prime ideal of $R$. Note that $e_{i_{0}}-m_{i_{0}} \neq 0$ since $e_{i_{0}}>f_{i_{0}}$. If $M$ is a maximal prime ideal of $R$ containing $A$, then there exists a $k, 1 \leqq k \leqq s$, such that $e_{k}-m_{k} \neq 0$ and $M_{k} \subseteq M$. Since $M_{k}$ is a maximal prime ideal of $R$, it follows that $M=M_{k}$. Now, $e_{k}-m_{k} \neq 0$ implies that $f_{k}-m_{k}=0$ which shows that $B \nsubseteq M_{k}=M$. Thus, if $M$ is a maximal prime ideal of $R$ containing $A, M$ does not contain $B$. It follows that $A+B$ is contained in no maximal prime ideal of $R$. Therefore, there exists a positive integer $\lambda$ such that $A+B=R^{\lambda}$ and $\left(x_{1}, x_{2}\right)=$ $P M_{1}^{m_{1}} \cdots M_{s}^{m_{s}}(A+B)=P M_{1}^{m_{1}} \cdots M_{s}^{m_{s}} R^{\lambda}=P M_{1}^{m_{1}} \cdots M_{s}^{m_{s}}$. By our choice of $m_{i}$, we have $e_{i} \geqq m_{i}$ for $1 \leqq i \leqq s$. But $e_{i_{0}}<f_{i_{0}}=m_{i_{0}}$ implies that

${ }^{3}$ The proof of Lemma 2 was suggested to the author by Professor Gilmer. 
$\delta-1 \geqq \sum_{i=1}^{s} m_{i} \geqq 0$.

Assume that we have chosen, as described above, $x_{1}, x_{2}, \cdots, x_{u}$ in $P$ such that $\left(x_{1}, \cdots, x_{u}\right)=P M_{1}^{v_{1}} \cdots M_{s}^{v_{s}}$ and $\delta-(u-1) \geqq \sum_{i=1}^{s} v_{i} \geqq 0$. Then by the above method, either $P=\left(x_{1}, \cdots, x_{u}\right)$ or there exists an $x_{u+1} \in P \backslash\left\{\left(x_{1}, \cdots, x_{u}\right) \cup\left(\bigcup_{i=1}^{n} P_{i}\right)\right\}$ such that

$$
\left(x_{1}, \cdots, x_{u}, x_{u+1}\right)=P M_{1}^{v_{1}^{\prime}} \cdots M_{s}^{v_{s}^{\prime}}
$$

where $v_{i}^{\prime} \in \omega_{0}$ and $\delta-(u+1-1) \geqq \sum_{i=1}^{s} v_{i}^{\prime} \geqq 0$. Since $\sum_{i=1}^{s} e_{i}$ is a finite positive number, there exists a positive integer $q$ and $x_{1}, \cdots, x_{q} \in P$ such that $P=\left(x_{1}, \cdots, x_{q}\right)$; that is, $P$ is a finitely generated ideal of $R$.

Case 2. $P^{2} \subset P$ and $P=R P$. Now, $P \nsubseteq\left\{P^{2} \cup\left(\bigcup_{i=1}^{n} P_{i}\right)\right\}$ by [2; Proposition 2, p. 70] so let $x_{1} \in P \backslash\left\{P^{2} \cup\left(\bigcup_{i=1}^{n} P_{i}\right)\right\}$. Then there exist prime ideals $M_{1}, \cdots, M_{s}$ of $R, e_{1}, \cdots, e_{s} \in \omega$, and $e_{s+1} \in \omega_{0}$ such that $\left(x_{1}\right)=P M_{1}^{e_{1}} \cdots M_{s}^{e_{s}} R^{e_{s+1}}=P M_{1}^{e_{1}} \cdots M_{s}^{e_{s}}$ since $P=R P$. If $P=\left(x_{1}\right)$ we are done. If $\left(x_{1}\right) \subset P$, then we can choose an

$$
x_{2} \in P \backslash\left\{\left(x_{1}\right) \cup P^{2} \cup\left(\bigcup_{i=1}^{n} P_{i}\right)\right\}
$$

by [2; Proposition 2, p. 70]. We now consider $\left(x_{1}, x_{2}\right)$ and the remainder of the proof of Case 2 is the same as the proof of Case 1. Thus, $P$ is a finitely generated ideal of $R$.

Case 3. $P^{2} \subset P$ and $R P \subset P$. Let $x \in P \backslash R P$. Then there exist prime ideals $M_{1}, \cdots, M_{s}$ of $R$ and $e_{1}, \cdots, e_{s+1} \in \omega_{0}$ such that $(x)=P M_{1}^{e_{1}} \cdots M_{s}^{e_{s}} R^{e_{s+1}} \nsubseteq R P$. Thus, $e_{i}=0$ for $1 \leqq i \leqq s+1$; that is, $P=(x)$.

Lemma 3. Each prime ideal of a general Z.P.I.-ring is finitely generated.

Proof. Let $R$ be a general Z.P.I.-ring.

Case 1. $R$ contains no proper prime ideal. If $R=R^{2}$, let $r \in R \backslash\{0\}$. Since $R$ is a general Z.P.I.-ring, there exists a positive integer $n$ such that $(r)=R^{n}=R$. If $R^{2} \subset R$, let $r \in R \backslash R^{2}$. Then $(r)=R$.

Case 2. $R$ contains a proper prime ideal. Let $M$ be a nonzero prime ideal of $R$. If $M$ is a minimal prime ideal of $R, M$ is finitely generated by Lemma 2. If $M$ is not a minimal prime ideal of $R$, the proof of Lemma 1 implies that there exists a minimal prime ideal $P$ of $R$ such that $P \subset M$. Thus, $R / P$ is Noetherian which implies that $M / P$ is a finitely generated ideal of $R / P$. Since $P$ is a finitely generated ideal of $R$, it follows that $M$ is a finitely generated ideal of $R$.

Thus, each prime ideal of $R$ is finitely generated.

Theorem 1. A general Z.P.I.-ring is Noetherian. 
Proof. Let $A$ be an ideal of $R$, a general Z.P.I.-ring. Then there exist prime ideals $P_{1}, \cdots, P_{n}$ of $R$ and positive integers $e_{1}, \cdots, e_{n}$ such that $A=P_{1}^{e_{1}} \cdots P_{n}^{e_{n}}$. Since each $P_{i}$ is finitely generated by Lemma 3 , it follows that $A$ is finitely generated. Thus, $R$ is Noetherian.

REMARK. Theorem 1 also follows from the fact that a ring $R$ is Noetherian if and only if each prime ideal of $R$ is finitely generated. [4; Th. 2].

ReSULT 1. If $Q$ is a P-primary ideal in a ring $R$ such that $Q$ can be represented as a finite product of prime ideals, then $Q$ is a power of $P$.

Proof. By hypothesis there exist distinct prime ideals $P_{1}, \cdots, P_{n}$ and positive integers $e_{1}, \cdots, e_{n}$ such that $Q=P_{1}^{e_{1}} \cdots P_{n}^{e_{n}}$. Since $Q=P_{1}^{e_{1}} \cdots P_{n}^{e_{n}} \leqq P, P_{i} \subset P$ for some $i$-say $i=1$. Now, $P=\sqrt{Q}=$ $P_{1} \cap \cdots \cap P_{n}$ which implies that $P \subseteq P_{i}$ for each $i$. Therefore, $P \subseteq P_{1} \subseteq P$; that is, $P_{1}=P$. We have that $Q=P^{e_{1}} P_{2}^{e_{2}} \cdots P_{n}^{e_{n}}$ where $P \subset P_{i}$ for $2 \leqq i \leqq n$. Since

$$
Q=P^{e_{1}}\left(P_{2}^{e_{2}} \cdots P_{n}^{e_{n}}\right) \subseteq Q
$$

and $P_{2}^{e_{2}} \cdots P_{n}^{e_{n}} \nsubseteq P$, it follows that $P^{e_{1}} \subseteq Q$. Hence, $Q=P^{e_{1}}$.

Definitions. Let $R$ be a ring. We say that $R$ has property $(\alpha)$, if each primary ideal of $R$ is a power of its (prime) radical [3]. If each ideal of $R$ is an intersection of a finite number of prime power ideals, we say that $R$ has property ( $\delta$ ) [3]. Finally, we say that $R$ satisfies property ( $\#)$ if $R$ is a ring without identity such that each nonzero ideal of $R$ is a power of $R$.

REMARK. If $R$ is a ring satisfying property ( $\sharp$ ), it follows that either $R$ is an integral domain in which $\left\{R^{i}\right\}_{i=1}^{\infty}$ is the collection of nonzero ideals of $R$ or $R$ is not an integral domain and $\left\{R, R^{2}, \cdots, R^{n}=(0)\right\}$ is the collection of all ideals of $R$ for some $n \in \omega$.

Corollary 1. A general Z.P.I.-ring has property $(\alpha)$.

Proof. This follows immediately from Result 1.

THEOREM 2. Structure theorem of a general Z.P.I.-ring. $A$ ring $R$ is a general Z.P.I.-ring if and only if $R$ has the following structure: 
(a) If $R=R^{2}$, then $R=R_{1} \oplus \cdots \oplus R_{n}$ where $R_{i}$ is either $a$ Dedekind domain or a special P.I.R. for $1 \leqq i \leqq n$.

(b) If $R \neq R^{2}$, then either $R=F \oplus T$ or $R=T$ where $F$ is a field and $T$ is a ring satisfying property (\#).

Proof. $(\rightarrow)$ If $R$ is a general Z.P.I.-ring, then $R$ is Noetherian and has property $(\alpha)$. Hence, [3; Corollary 6] implies that $(\delta)$ holds in $R$. If $R=R^{2}$, then $R$ contains an identity by [5; Corollary 2]. Therefore, [1; Th. 1] implies that part (a) holds. If $R \neq R^{2}$, then by (BG) $R=F_{1} \oplus \cdots \oplus F_{u} \oplus T$ where each $F_{i}$ is a field and $T$ is a nonzero ring satisfying property (\#). Using a contrapositive argument, we show that $u \nsupseteq 2$.

Assume that $u \geqq 2$. We show that $R$ is not a general Z.P.I.ring. Since $u \geqq 2$, it is clear that $T$ is an ideal of $R$ that is not prime. The prime ideals of $R$ containing $T$ are $R$ and

$$
P_{i}=F_{1} \oplus \cdots \oplus F_{i-1} \oplus(0) \oplus F_{i+1} \oplus \cdots \oplus F_{u} \oplus T
$$

for $1 \leqq i \leqq u$ where $T \subset P_{i}$ for each $i$. Now

$$
\begin{aligned}
& P_{i} P_{j} \\
= & F_{1} \oplus \cdots \oplus F_{i-1} \oplus(0) \oplus F_{i+1} \oplus \cdots \oplus F_{j-1} \oplus(0) \oplus F_{j+1} \oplus \cdots \oplus F_{u} \oplus T^{2}, \\
& R P_{i}=F_{1} \oplus \cdots \oplus F_{i-1} \oplus(0) \oplus F_{i+1} \oplus \cdots \oplus F_{u} \oplus T^{2},
\end{aligned}
$$

and $R^{2}=F_{1} \oplus \cdots \oplus F_{u} \oplus T^{2}$. Since $T^{2} \subset T$, it follows that $T \nsubseteq P_{i} P_{j}$, $T \nsubseteq R P_{i}$, and $T \nsubseteq R^{2}$ for $1 \leqq i, j \leqq u$. Thus, $T$ cannot be represented as a finite product of prime ideals of $R$; that is, $R$ is not a general Z.P.I.-ring. Therefore, if $R$ is a general Z.P.I.-ring, $u \nsupseteq 2$; that is, $R=F_{1} \oplus T$ or $R=T$ where $F_{1}$ is a field and $T$ is a ring satisfying property (\#).

$(\leftarrow)$ If $R$ is a direct sum of finitely many Dedekind domains and special P.I.R.'s $R$ is a general Z.P.I.-ring by [1; Th. 1]. If $R=T$ where $T$ is a ring satisfying property $(\#)$, then $R$ is clearly a general Z.P.I.-ring. If $R=F \oplus T$ where $F$ is a field and $T$ is a ring satisfying property (\#), then $\left\{F \oplus T^{i}, T^{i},(0): i \in \omega\right\}$ is the collection of ideals of $R$. It follows that each ideal of $R$ is a finite product of prime ideals. Therefore, if $R$ satisfies either (a) or (b), $R$ is a general Z.P.I.-ring.

3. Necessary and sufficient conditions on a general Z.I.P.ring. In this section we again use results of Butts and Gilmer in [3] to derive several necessary and sufficient conditions for a ring to be a general Z.P.I.-ring.

Definition. Let $A$ be an ideal of a ring $R$. We say that $A$ 
is simple if there exist no ideals properly between $A$ and $A^{2}$. To avoid conflicts with other definitions of a simple ring we say in case $A=R$ that $R$ satisfies property $S$.

Lemma 4. Let $A$ be an ideal of a Noetherian ring $R$. If $B=\bigcap_{i=1}^{\infty} A^{i}$, then $A B=B$.

Proof. See $\left[15 ; L_{1}\right]$.

Lemma 5. If $A$ is a genuine ideal of a Noetherian domain $D$, then $\bigcap_{i=1}^{\infty} A^{i}=(0)$.

Proof. Let $K$ be the quotient field of $D$ and let $D^{*}=D[e]$ where $e$ is the identity of $K$. Then $D^{*}$ is Noetherian by [5; Th. 1], and since $A$ is also an ideal of $D^{*},[16$; Corollary 1, p. 216] shows that $\bigcap_{i=1}^{\infty} A^{i}=(0)$.

Lemma 6. Let $A$ be a simple ideal of a ring $R$. Then for each $i \in \omega$ there are no ideals properly between $A^{i}$ and $A^{i+1}$. Further, the only ideals between $A$ and $A^{n}$ for $n \in \omega$ are $A, A^{2}, \cdots, A^{n}$.

Proof. See [7; Lemma 3].

Lemma 7. Let $A$ be a proper simple ideal of a Noetherian ring R. If there exists a prime ideal $P$ of $R$ such that $(0) \subset P \subset A \subset R$, $P$ is unique and $P=\bigcap_{i=1}^{\infty} A^{i}$. Also, if $Q$ is a P-primary ideal of $R, Q=P$.

Proof. We first show by an inductive argument that $P \subset A^{i}$ for each $i \in \omega$. By hypothesis $P \subset A$. Assume that $P \subset A^{k}$ for some $k \in \omega$. Since $A / P$ is a proper ideal of $R / P$, a Noetherian integral domain, $A^{k} / P \supset\left(A^{k} / P\right)(A / P)=\left(A^{k+1}+P\right) / P \supset P / P$ by [5; Corollary 1] which shows that $A^{k} \supset A^{k+1}+P \supseteqq A^{k+1}$. Therefore, $A^{k+1}+P=A^{k+1}$. Since $A^{k+1}+P \supset P$, it follows that $P \subset A^{k+1}$. Thus, $P \subset A^{i}$ for each $i \in \omega$.

We now show that $P=\bigcap_{i=1}^{\infty} A^{i}$. Since $A / P$ is a proper ideal of a Noetherian domain, $P / P=\bigcap_{i=1}^{\infty}(A / P)^{i}$ by Lemma 5 . Also, since $\bigcap_{i=1}^{\infty}(A / P)^{i}=\bigcap_{i=1}^{\infty}\left(\left(A^{i}+P\right) / P\right)=\bigcap_{i=1}^{\infty}\left(A^{2} / P\right)=\left(\bigcap_{i=1}^{\infty} A^{i}\right) / P$, it follows that $P=\bigcap_{i=1}^{\infty} A^{i}$.

Finally, we show that if $Q$ is a $P$-primary ideal of $R$, then $Q=P$. Lemma 4 shows that $P=A\left(\bigcap_{i=1}^{\infty} A^{i}\right)=A P$. There exists an $a \in A$ such that $a p=p$ for each $p \in P$ by [5; Corollary 1]; that is, $a p-p=0$ for each $p \in P$. If $x \in R \backslash A$, then $p(a x-x)=a p x-p x=$ $0 \in Q$ for each $p \in P$. Since $x \notin A, a x-x \notin A$ which shows that $a x-x \notin P$. Thus, $p \in Q$ for each $p \in P$ since $p(a x-x) \in Q$ for each 
$p \in P, a x-x \notin P$, and $Q$ is a $P$-primary ideal of $R$. Thus, $P \subseteq Q$ which shows that $Q=P$.

THEOREM 3. Let $R$ be a ring.

(A) If $R$ contains an identity, then $R$ is a general Z.P.I.-ring if and only if $R$ satisfies the following two conditions:

(1) $R$ is Noetherian.

(2) Each maximal ideal of $R$ is simple.

(B) If $R$ does not contain an identity and $R$ contains a proper prime ideal, then $R$ is a general Z.P.I.-ring if and only if $R$ satisfies the following four conditions:

(1) $R$ is Noetherian.

(2) $R$ satisfies property $S$.

(3) Each maximal prime ideal of $R$ is simple.

(4) $\bigcap_{i=1}^{\infty} R^{i}$ is a field.

(C) If $R$ does not contain an identity and $R$ contains no proper prime ideal, then $R$ is a general Z.P.I.-ring if and only if $R$ satisfies the following two conditions:

(1) $R$ is Noetherian.

(2) $R$ satisfies property $S$.

Proof of (A). Part (A) follows immediately from [1; Th. 5].

Proof of $(\mathrm{B}) . \quad(\rightarrow)$ Assume that $R$ is a general Z.P.I.-ring. Then $R$ is Noetherian by Theorem 1 . Since $R$ contains a proper prime ideal, Theorem 2 shows that $R=F \oplus T$ where $F$ is a field and $T$ is a ring satisfying property ( $\#)$. Hence, $R$ clearly satisfies property $S$. If $T$ is a domain, then $F$ and $T$ are the maximal prime ideals of $R$. If $T$ is not a domain, then $T$ is the maximal prime ideal of $R$. It follows that each maximal prime ideal of $R$ is simple. Finally, $\bigcap_{i=1}^{\infty} R^{i}=\bigcap_{i=1}^{\infty}(F \oplus T)^{i}=F$, a field.

$(\leftarrow)$ Assume that conditions (1), (2), (3), and (4) hold. Let $Q$ be a $P$-primary ideal of $R$. If $P=R$ or if $P$ is a maximal prime ideal of $R$, there exists an integer $n$ such that $P^{n} \cong Q$ since $R$ is Noetherian. Hence Lemma 6 shows that there exists an integer $k$ such that $Q=P^{k}$. If $P$ is a proper nonmaximal prime ideal of $R$, there exists a maximal prime ideal $M$ of $R$ such that $P \subset M \subset R$, and it follows from Lemma 7 that $Q=P$. Thus, $R$ is a Noetherian ring having property $(\alpha)$ which shows that $(\delta)$ holds in $R$. [3; Corollary 6]. Therefore, by (BG) $R=F_{1} \oplus \cdots \oplus F_{m} \oplus T$ where each $F_{i}$ is a field and $T$ satisfies property (\#). Since $R$ contains a proper prime ideal, $m \geqq 1$; condition (4) implies that $m \ngtr 1$. Hence $R=F_{1} \oplus T$ which implies that $R$ is a general Z.P.I.-ring. 
Proof of $(\mathrm{C}) . \quad(\rightarrow)$ If $R$ is a general Z.P.I.-ring containing no proper prime ideal, then $R=T$ where $T$ is a ring satisfying property $(\#)$, Hence, $R$ is Noetherian and satisfies property $S$.

$(\leftarrow)$ Assume that conditions (1) and (2) hold. Since $R$ is Noetherian and since $R$ is the only nonzero prime ideal in $R, R$ has property $(\alpha)$. Thus, $R$ is a general Z.P.I.-ring by an argument similar to that given in part (B) above.

Lemma 8. A ring $R$ has property ( $\delta$ ) if and only if $R$ satisfies the following three conditions:

(1) $R$ is Noetherian.

(2) $R$ satisfies property $S$.

(3) Each maximal prime ideal of $R$ is simple.

Proof. $(\rightarrow)$ Assume that $R$ has property $(\delta)$. If $R=R^{2}$, [3; Th. 11] implies that $R$ is a general Z.P.I.-ring. Therefore, (1), (2), and (3) hold by Theorem 3. If $R \neq R^{2}$, then [3; Th. 12] implies that $R$ is Noetherian. From (BG) we have that $R=F_{1} \oplus \cdots \oplus F_{m} \oplus T$ where each $F_{i}$ is a field and $T$ satisfies property (\#). It follows from the representation of $R$, that (2) and (3) hold.

$(\leftarrow)$ We showed in the proof of Theorem 3 (B) that if (1), (2), and (3) hold in a ring $R$, then $(\delta)$ holds in $R$.

Lemma 9. In a Noetherian ring $R$, property $(\alpha)$ is equivalent to the following two conditions:

(2) $R$ satisfies property $S$.

(3) Each maximal prime ideal of $R$ is simple.

Proof. This follows immediately from Lemma 8 and [3; Corollary 6].

THEOREM 4. If $R$ is a ring with identity, $R$ is a general Z.P.I.-ring if and only if $R$ is Noetherian and $(\alpha)$ holds in $R$.

Proof. The necessity follows from Theorem 1 and Corollary 1 and the sufficiency follows from [3; Corollary 6 and Th. 11].

COROLlary 2. Let $R$ be a ring without identity.

(A) If $R$ contains a proper prime ideal, then $R$ is a general Z.P.I.-ring if and only if $R$ satisfies the following three conditions:

(1) $R$ is Noetherian.

(2') $(\alpha)$ holds in $R$.

(4) $\bigcap_{i=1}^{\infty} R^{i}$ is a field.

(B) If $R$ contains no proper prime ideal, then $R$ is a general 
Z.P.I.-ring if and only if $R$ satisfies the following two conditions:

(1) $R$ is Noetherian.

(2') ( $\alpha$ ) holds in $R$.

Proof. This follows immediately from Theorem 3 and Lemma 9.

\section{REFERENCES}

1. Keizo Asano, Über kommutative Ringe, in denen jedes Ideal als Produkt von Primidealen darstellbar ist, J. Math. Soc. Japan 1 (1951), 82-90.

2. N. Bourbaki, Éleménts de Mathématique, Algèbre Commutative, Chap. 7, Paris, 1961.

3. H. S. Butts and Robert W. Gilmer, Jr., Primary ideals and prime power ideals, Canad. J. Math. 18 (1966), 1183-1195.

4. I. S. Cohen, Commutative rings with restricted minimum condition, Duke Math.

J. 17 (1950), 27-42.

5. Robert W. Gilmer, Jr., Eleven nonequivalent conditions on a commutative ring, Nagoya Math. J. 26(1966), 183-194.

6. - On a classical theorem of Noether in ideal theory, Pacific J. Math. 13 (1963), 579-583.

7. Robert W. Gilmer, Jr., and Joe Leonard Mott, Multiplication rings as rings in which ideals with prime radical are primary, Trans. Amer. Math. Soc. 114 (1965), 40-52.

8. Wolfgang Krull, Idealtheorie, New York, 1948.

9. —— Über den Aufbau des Nullideals in ganz abgeschlossenen Ringen mit Teilerkettensatz, Math. Ann. 102 (1926). 363-369.

10. Keizi Kubo, Über die Noetherschen fünf Axiome in kommutativen Ringen, J. Sci. Hiroshima Univ. (A) 10 (1940), 77-84.

11. Shinziro Mori, Allgemeine Z.P.I.-Ringe, J. Sci. Hiroshima Univ. (A) 10 (1940), 117-136.

12. - Axiomatische Begründung des Multiplikationringe, J. Sci. Hiroshima Univ. (A) 3 (1932), 45-59.

13. E. Noether, Abstrakter Aufbau der Idealtheorie in algebraischen Zahl-und Funktionenkörpern, Math. Ann. 96 (1926), 36-61.

14. M. Sono, On congruences, II, Mem. Coll. Sci. Kyoto Univ. 3 (1918), 113.

15. Michio Yoshida and Motoyoshi Sakuma, The intersection theorem on Noetherian rings, J. Sci. Hiroshima Univ. (A) 17 (1954), 317-320.

16. Oscar Zariski and Pierre Samuel, Commutative algebra, Vol. I, Princeton, 1958.

17. — Commutative algebra, Vol. II, Princeton, 1960.

Received November 5, 1968. This paper is a portion of the author's doctoral dissertation, which was written under the direction of Professor Robert W. Gilmer Jr., at The Florida State University.

The Florida State University

Tallahasse, Florida 


\section{PACIFIC JOURNAL OF MATHEMATICS}

\section{EDITORS}

H. ROYDEN

Stanford University

Stanford, California

\section{RichaRd PIERCE}

University of Washington Seattle, Washington 98105
J. DugundJI

Department of Mathematics

University of Southern California

Los Angeles, California 90007

BASIL GORDON

University of California

Los Angeles, California 90024

\section{ASSOCIATE EDITORS}
E. F. BECKENBACH
B. H. NeUMANN
F. WOLF
K. YOSHIDA

\section{SUPPORTING INSTITUTIONS}

UNIVERSITY OF BRITISH COLUMBIA CALIFORNIA INSTITUTE OF TECHNOLOGY

UNIVERSITY OF CALIFORNIA

MONTANA STATE UNIVERSITY

UNIVERSITY OF NEVADA

NEW MEXICO STATE UNIVERSITY

OREGON STATE UNIVERSITY

UNIVERSITY OF OREGON

OSAKA UNIVERSITY

UNIVERSITY OF SOUTHERN CALIFORNIA

\author{
STANFORD UNIVERSITY \\ UNIVERSITY OF TOKYO \\ UNIVERSITY OF UTAH \\ WASHINGTON STATE UNIVERSITY \\ UNIVERSITY OF WASHINGTON \\ AMERICAN MATHEMATICAL SOCIETY \\ CHEVRON RESEARCH CORPORATION \\ TRW SYSTEMS \\ NAVAL WEAPONS CENTER
}

The Supporting Institutions listed above contribute to the cost of publication of this Journal, but they are not owners or publishers and have no responsibility for its content or policies.

Mathematical papers intended for publication in the Pacific Journal of Mathematics should be in typed form or offset-reproduced, double spaced with large margins. Underline Greek letters in red, German in green, and script in blue. The first paragraph or two must be capable of being used separately as a synopsis of the entire paper. It should not contain references to the bibliography. Manuscripts, in duplicate if possible, may be sent to any one of the four editors. Please classify according to the scheme of Math. Rev. 36, 1539-1546. All other communications to the editors should be addressed to the managing editor, Richard Arens, University of California, Los Angeles, California, 90024.

50 reprints are provided free for each article; additional copies may be obtained at cost in multiples of 50 .

The Pacific Journal of Mathematics is published monthly. Effective with Volume 16 the price per volume (3 numbers) is $\$ 8.00$; single issues, $\$ 3.00$. Special price for current issues to individual faculty members of supporting institutions and to individual members of the American Mathematical Society: $\$ 4.00$ per volume; single issues $\$ 1.50$. Back numbers are available.

Subscriptions, orders for back numbers, and changes of address should be sent to Pacific Journal of Mathematics, 103 Highland Boulevard, Berkeley, California, 94708.

PUBLISHED BY PACIFIC JOURNAL OF MATHEMATICS, A NON-PROFIT CORPORATION

Printed at Kokusai Bunken Insatsusha (International Academic Printing Co., Ltd.), 7-17. Fujimi 2-chome, Chiyoda-ku, Tokyo, Japan. 


\section{Pacific Journal of Mathematics}

\section{Vol. 30, No. $3 \quad$ November, 1969}

Willard Ellis Baxter, Topological rings with property $(Y) \ldots \ldots \ldots \ldots . \ldots 5$

Sterling K. Berberian, Note on some spectral inequalities of $C . R$.

Putnam ..................................... 573

David Theodore Brown, Galois theory for Banach algebras . ........... 577

Dennis K. Burke and R. A. Stoltenberg, A note on p-spaces and Moore spaces ........................................ 601

Rafael Van Severen Chacon and Stephen Allan McGrath, Estimates of positive contractions....................................... 609

Rene Felix Dennemeyer, Conjugate surfaces for multiple integral problems in the calculus of variations ........................... 621

Edwin O. Elliott, Measures on countable product spaces.............. 639

John Moss Grover, Covering groups of groups of Lie type .............. 645

Charles Lemuel Hagopian, Concerning semi-local-connectedness and cutting in nonlocally connected continua .................. 657

Velmer B. Headley, A monotonicity principle for eigenvalues ........... 663

John Joseph Hutchinson, Intrinsic extensions of rings . . . . . . . . . . . . . 669

Harold H. Johnson, Determination of hyperbolicity by partial

prolongations .................................. 679

Tilla Weinstein, Holomorphic quadratic differentials on surfaces in $E^{3} \ldots 697$

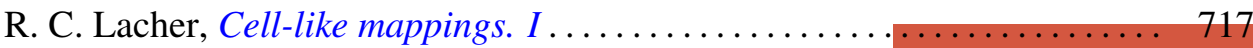

Roger McCann, A classification of centers

Curtis L. Outlaw, Mean value iteration of nonexpansive mappings in a

Banach space...

Allan C. Peterson, Distribution of zeros of solutions of a fourth order

differential equation.

Bhalchandra B. Phadke, Polyhedron inequality and strict convexity .. 765 Jack Wyndall Rogers Jr., On universal tree-like continua .

Edgar Andrews Rutter, Two characterizations of quasi-Frobenius rings

G. Sankaranarayanan and C. Suyambulingom, Some renewal theorems concerning a sequence of correlated random variables...

Joel E. Schneider, A note on the theory of primes........ . .

Richard Peter Stanley, Zero square rings .................

Edward D. Tymchatyn, The 2-cell as a partially ordered space

Craig A. Wood, On general Z.P.I.-rings................ 